\title{
Surveying for Dwarf Galaxies Within Voids FN2 and FN8
}

\author{
Stephen McNeil ${ }^{1}$, Chris Draper ${ }^{2}$ and J. Ward Moody ${ }^{3}$ \\ ${ }^{1}$ Dept. of Physics, 118 ROM \\ Brigham Young University Idaho, Rexburg, ID 83460-0520 USA \\ email: mcneils@byui.edu \\ ${ }^{2}$ Dept. of Physics, MS 179 \\ Utah Valley University, Orem, UT 84058 USA \\ email: drapechr@uvu.edu \\ ${ }^{3}$ Dept. of Physics and Astronomy, N283 ESC \\ Brigham Young University, Provo, UT 86402-4666 USA \\ email: jmoody@byu.edu
}

\begin{abstract}
The presence or absence of dwarf galaxies with $\mathrm{M}_{r^{\prime}}>-14$ in low-density volumes correlates with dark matter halos and how they affect galaxy formation. We are conducting a redshifted $\mathrm{H} \alpha$ imaging survey for dwarf galaxies with $\mathrm{M}_{r^{\prime}}>-13$ in the heart of the well-defined voids FN2 and FN8 using the KPNO 4m Mayall telescope and Mosaic Imager. These data have furnished over 600 strong candidates in a four square degree area. Follow-up spectra finding none of these candidates to be within the void volumes will constrain the dwarf population there to be 2 to $8 \%$ of the cosmic mean. Conversely, finding even one $\mathrm{H} \alpha$ dwarf in the void heart will challenge several otherwise successful theories of large-scale structure formation.
\end{abstract}

Keywords. large-scale structure of universe, galaxies: distances and redshifts, galaxies: dwarf

\section{Introduction}

Lambda cold dark matter $(\Lambda \mathrm{CDM})$ models predict the existence of many low-mass dark matter halos in voids (e.g. Dekel \& Silk 1986, Peebles 2001, Hoffman, Silk, \& Wyse 1992, Tikhanov \& Klypin 2009.) If galaxy formation has proceeded in these halos, then voids should have a population of dwarf galaxies in their interior (e.g. Tikhanov \& Klypin 2009). But studies like Hoyle et al. (2005), do not find them. This problem was termed the "void phenomenon" by Peebles (2001).

To date, void centers have not been systematically searched in large-area optical spectroscopic surveys to levels faint enough to find such dwarfs. For example, the SDSS spectroscopic limit of $r^{\prime}>17.77$ is sufficient to locate theoretically interesting dwarf galaxies with $\mathrm{M}_{r^{\prime}}=-14$ only out to a $\mathrm{cz}$ of $1700 \mathrm{~km} / \mathrm{sec}$. Well defined void centers are not reached until 4,000 to $6,000 \mathrm{~km} / \mathrm{sec}$. Therefore the work of ruling out the existence of dwarf galaxies in the centers of voids through such surveys has yet to be accomplished.

\section{Technique}

In February 2013 we used the KPNO 4m Mayall telescope+Mosaic Imager to examine the heart of voids FN2 $(\mathrm{RA}=3 \mathrm{~h} 45 \mathrm{~m}, \mathrm{DEC}=+18 \mathrm{deg}, \mathrm{cz}=4550 \mathrm{~km} / \mathrm{sec}$, diam. $=$ $5070 \mathrm{~km} / \mathrm{sec})$ and FN8 $(\mathrm{RA}=12 \mathrm{~h} 32 \mathrm{~m}, \mathrm{DEC}=+71.3 \mathrm{deg} \mathrm{cz}=4980 \mathrm{~km} / \mathrm{sec}$, diam. $=$ $3660 \mathrm{~km} / \mathrm{sec}$ ) from Foster \& Nelson (2009). Exposures were through three redshifted $\mathrm{H} \alpha$ filters: $\mathrm{H} 8(\Delta \lambda 661.2-669.0 \mathrm{~nm}), \mathrm{H} 12(\Delta \lambda 665.1-673.0 \mathrm{~nm})$, and $\mathrm{H} 16(\Delta \lambda 668.5-676.3 \mathrm{~nm})$. 


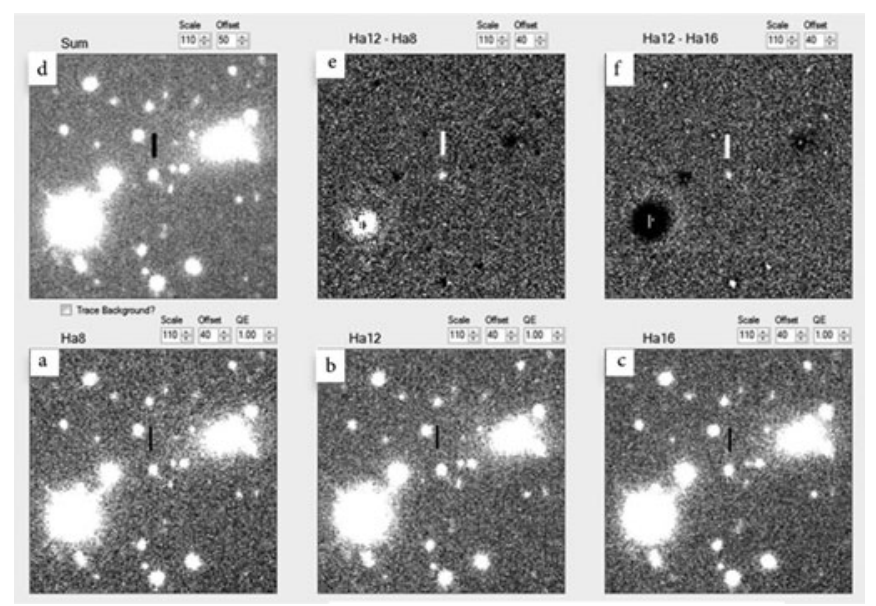

Figure 1. A crowded field showing images through filters a) H8, b) H12, and c) H16. Image d) is the sum of all filters, image e) is H12-H8, and image f) is H12-H16. The marked object stands out in both e) and f) because it has an emission line centered in filter H12. If the emission is $\mathrm{H} \alpha$, it is a dwarf galaxy in the void center.

Seventeen fields (thirteen in FN8 and four in FN2) covering four square degrees were imaged. Data were summed over all filters for each field and over 400,000 objects were selected from the summed frames using Source Extractor (Bertin \& Arnouts 1996). Each object was categorized by its value of H12/H8 vs H12/H16. Objects at the distance of the void center having $\mathrm{H} \alpha$ emission greater than $5.0 \mathrm{~nm}$ have both $\mathrm{H} 12 / \mathrm{H} 8$ and $\mathrm{H} 12 / \mathrm{H} 16$ index values greater than 1.1 while objects with no emission have values close to 1.0. Candidates for being inside the voids (over 600) were collected and examined by eye using our own software package Compare Images to reject flaws (see Fig. 1).

\section{Discussion}

The summed signal for an object with $\mathrm{M}_{r^{\prime}}=-13$ is a three to five sigma detection for all fields. At this threshold we are finding many more candidates than expected for the void density and LMF distribution of Hoyle et al. (2005). This is in part from detecting objects with strong $[\mathrm{OIII}] \lambda 5007$ in a supercluster at $\mathrm{z} \sim 0.3$ as judged from maps and object sizes and morphologies. This result is encouraging since it provides a critical measure of the depth of the survey technique and the detection limit of void dwarfs.

The search technique works well and we will be able to find dwarfs at the void center with $\mathrm{M}_{r^{\prime}}>-13$ if they are present. Given the large contamination from the backside, our data are consistent with completely empty voids, but they are also consistent with dwarf galaxies being present. Follow-up spectra alone will determine which candidates are dwarfs and which are not and will resolve the observational side of the void phenomenon.

\section{References}

Bertin, E. \& Arnouts, S. 1996, A\&AS, 317, 393

Dekel, A. \& Silk, J. 1986, ApJ, 303, 39

Foster, C. \& Nelson, L. A. 2009, ApJ, 699, 1252

Hoffman, Y., Silk, J., \& Wyse, R. F. G. 1992, ApJ, 388, L13

Hoyle, F., Rojas, R. R., Vogeley, M. S., \& Brinkmann, J. 2005, ApJ, 620, 618

Peebles, P. J. E. 2001, ApJ, 557, 495

Tikhonov, A. V. \& Klypin, A. 2009, MNRAS, 395, 1915 